\title{
TECNOLOGÍA DE LA INFORMACIÓN Y COMUNICACIÓN COMO HERRAMIENTA PARA LA TOMA DE DECISIONES EN LAS INSTITUCIONES EDUCATIVAS EN SANTA MARTA, COLOMBIA
}

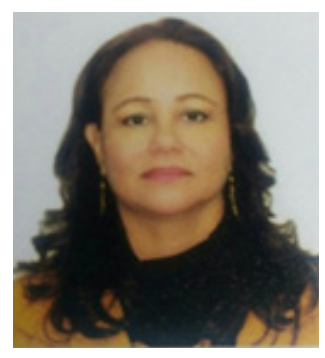

\author{
Elvia Lacera Padilla* \\ Institución Educativa Distrital Simón Rodriguez., Colombia \\ elvialacera@gmail.com
}

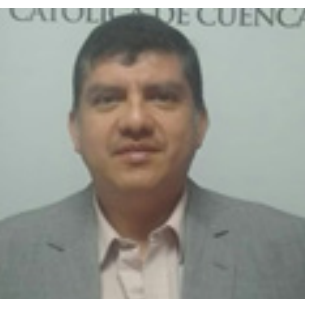

\author{
Marcos Orellana Parra** \\ Universidad Católica de Cuencas. Ecuador. \\ morellanap@ucacue.edu.ec
}

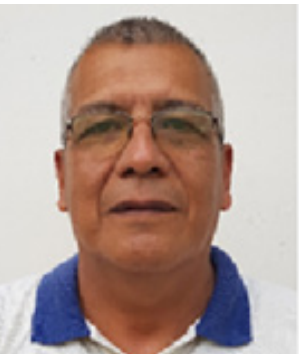

\section{Gonzalo Gustavo Cortez*** \\ Universidad Laica Eloy Alfaro de Manabí, Ecuador \\ Gustavo.cortez@uleam.edu.ec}

\section{RESUMEN}

El objetivo fue analizar la tecnología de la información y la comunicación (TIC) como herramienta para la toma de decisiones en las instituciones educativas en Santa Marta. La investigación fue descriptiva con diseño de campo, transeccional y no experimental. La población estuvo conformada por 7 unidades educativas públicas básicas primaria de la localidad 2. Los informantes fueron 7 rectores y 86 docentes. Se diseñaron dos instrumentos con escala tipo Lickert. Fueron sometidos a validez y confiabilidad a través del método Alfa de Cronbach obteniéndose una confiabilidad de 0,852 para los rectores y 0,994 para los docentes. Se demostró para la variable TIC que la mayor frecuencia de las respuestas, tanto de los rectores (35\%) como docentes $(34,6 \%)$, estuvo enmarcada en casi siempre. En cuanto a la toma de decisiones, la alternativa siempre tuvo el mayor porcentaje (34,6\%) para el grupo de rectores y para los docentes las respuestas estuvo orientada hacia casi siempre $(41,2 \%)$. Se demuestra presencia de ambas variable: TIC alcanzó una media de 3.88 y toma de decisiones, 3.92; sin embargo están muy cercanos al extremo inferior de la media ponderada, lo cual alerta a prestar especial atención ya que puede ser susceptible a descender a valores inferiores. 
Palabras clave: Tecnología de la información y comunicación, toma de decisiones, instituciones educativas, Santa Marta, Colombia.

\title{
INFORMATION AND COMMUNICATION TECHNOLOGY AS A TOOL FOR DECISION-MAKING IN EDUCATIONAL INSTITUTIONS, IN SANTA MARTA, COLOMBIA.
}

\begin{abstract}
The objective was to analyze information and communication technology (ICT) as a tool for decision making in educational institutions in Santa Marta. The research was descriptive with field design, transectional and non-experimental. The population consisted of 7 primary public basic education units of the locality 2. The informants were 7 rectors and 86 teachers. Two instruments with a Lickert type scale were designed. They were subjected to validity and reliability through the Cronbach's Alpha method, obtaining a reliability of 0.852 for the rectors and 0.994 for the teachers. It was shown for the ICT variable that the highest frequency of responses, both from the rectors (35\%) and teachers (34.6\%), was almost always framed. Regarding decision making, the alternative always had the highest percentage $(34.6 \%)$ for the group of rectors and for teachers the answers were oriented towards almost always (41.2\%). The presence of both variables is demonstrated: TIC reached an average of 3.88 and decision making, 3.92; however, they are very close to the lower end of the weighted average, which warns to pay special attention because it may be susceptible to descend to lower values.
\end{abstract}

Keywords: Information and communication technology, decision making, educational institutions, Santa Marta, Colombia.

\section{INTRODUCCIÓN}

En la actualidad existe un reconocimiento del papel central que la educación desempeña en los procesos de desarrollo, este papel se relaciona con la capacidad para afrontar los desafíos planteados por la revolución científico-tecnológica, para ponerse al día con la transformación productiva que dicha revolución implica, para resolver problemas sociales y para consolidar los procesos educacionales.

Al respecto, la UNESCO (2009), señala que la educación tiene que adaptarse en todo momento a los cambios de la sociedad, sin dejar de transmitir por ello el saber adquirido, los principios y los frutos de la experiencia. Lo anterior conlleva a que cada vez

\footnotetext{
*Magister en Educación. Mención Gerencia de la Organizaciones Educativas. Licenciada en Lenguas Modernas. Docente de la Institución Educativa Distrital Simón Rodriguez.

**Magister en Educación Informática. Ingeniero en Electrónica y Telecomunicaciones. Docente de las Carreras de: Ingeniería en Tecnologías de la Información y Contabilidad y Auditoría. Universidad Católica de Cuencas. Ecuador.

***Magister en Docencia Universitaria. Abogado. Especialista en derecho penal. Docente de la Universidad Laica Eloy Alfaro de Manabí, Ecuador, Colombia.
}

ORATORES ISSN Impreso: 2644-3988 ISSN Electrónico: L-2644-3988 Año 6. Número 9. Diciembre 2018 - Mayo 2019 
se proyecten nuevas y mayores expectativas en ese ámbito, exigiéndosele al docente más tareas y responsabilidades en su quehacer con el propósito de que el proceso enseñanza aprendizaje, incida en la formación integral del estudiante de manera significativa.

Para que lo expresado se materialice, se requiere contar con herramientas de apoyo a la docencia. En este sentido, los directivos y docentes se deben adecuar a las nuevas tecnologías de información y comunicación (TIC), como estrategia dirigida a mejorar los cambios en el ámbito del quehacer educativo, dependiendo de las particularidades regionales y la concepción educativa implantada.

Desde esta perspectiva, los cambios educativos están orientados al uso de las nuevas tecnologías de la información y comunicación (TIC) en el proceso formativo, debido a que estas herramientas representan para la sociedad un medio comunicativo por excelencia, que permite al estudiante estar actualizado en cuanto a lo que sucede en el mundo, por lo tanto puede generar conocimientos significativos para su desarrollo integral.

En este sentido, las denominadas Tecnologías de la Comunicación e Información (TIC), según Aguerrondo (2006) son herramientas teórico conceptuales, que procesan, almacenan, sintetizan, recuperan y presentan información de la forma más variada. Entendiéndose que estas herramientas de comunicación son avances tecnológicos para obtener, guardar y presentar información en cualquier ámbito de la vida, por lo que no pueden quedar aisladas del sistema educativo.

Por lo tanto, las TIC imprimen nuevas orientaciones en el discurso y la práctica educativa, ya que aportan recursos pero además estrategias de organización visual, mental y cognitiva, que potencian así como también consolidan la adquisición de competencias en las diferentes áreas del conocimiento, siempre que sean ajustadas a las condiciones y características de cada una de ellas.

Igualmente, estas herramientas brindan la posibilidad de desarrollar un proceso de comunicación organizacional innovador, donde los directivos puedan reducir los espacios y momentos de la organización gerencial y el docente pueda desarrollar sus clases facilitando la adquisición de aprendizajes significativos en los estudiantes, permitiendo así la adquisición de conocimientos perdurables en el tiempo, sin olvidar que brinda la oportunidad de mejorar la comunicación entre los miembros de la institución.

Por consiguiente, es conveniente la necesidad de establecer instituciones educativas dinámicas, con nuevas maneras de hacer las cosas, las cuales ostenten una filosofía de gestión con un norte claro, porque en esa medida será su proyección hacia el logro de sus objetivos, es por tales razones que; la toma de decisiones adquiere un lugar importante dentro de la gestión de las mismas. 
De allí pues, que en el mundo de hoy las organizaciones requieren de profesionales abiertos al cambio, con disposición a enfrentar retos, desafíos, no de jefes dedicados a dar órdenes, tomar todas las decisiones y con frecuencia denotando una arrogancia exagerada, pensando siempre en tener la razón, siendo los demás limitados en todos los aspectos.

Es por ello, que es necesario precisar, que en las instituciones educativas al aplicar decisiones adecuadas se puede disponer de mayor tiempo para otras tareas, tales como evaluar misión, visión, objetivos, metas, asimismo se posibilita estar atento a los cambios del entorno para responder rápidamente a las nuevas necesidades y expectativas organizacionales, lo cual redundará en la eficiencia organizacional.

En este sentido, se podría decir que las tecnologías de información y comunicación, más que herramientas generadoras de productos finales, son procesos científicos cuyo principal objetivo es la generación de conocimientos, que a la postre incidirán en los modos de vida de las sociedades, no sólo en un ámbito técnico o especializado, sino principalmente en la creación de nuevas oportunidades para la toma decisiones del gerente educativo y convivencia institucional.

A este respecto, América Latina, de acuerdo a los planteamientos de Patru y Semenov (2005), se encuentra en la actualidad en un proceso donde los directivos y docentes deben vencer la resistencia al uso de las tecnologías de la información y comunicación en las actividades gerenciales educativas. Esto se hace evidente en situaciones como: poco desempeño en el uso de las tecnologías, resistencia a los cambios, apatía, malestar laboral, saboteo a las gestiones de cambios, abandono laboral, entre otros síntomas que evidencian resistencia a los cambios educativos.

Es por esta razón que particularmente el uso de esta herramienta se hace evidente en las desigualdades entre los individuos que dominan las TIC y quienes no las dominan, aspectos que causan descontentos dentro de la misma organización. Haciendo un análisis de la situación descrita y en concordancia con lo que se presenta en América Latina, se podrían acrecentar los problemas organizacionales; y por ende, una baja en la calidad educativa pues, se quedará rezagada en comparación a los estándares educativos mundiales.

De esta realidad no escapa Colombia, país que vive en los actuales momentos una profunda reestructuración social donde la calidad de la educación; aunado a los procesos gerenciales ha disminuido notablemente, como se puede evidenciar en aspectos muy notorios para la mayoría de quienes trabajan en el campo educativo, entre los que destacan: desinterés por los estudios, poca efectividad en los procesos gerenciales, poca creatividad en el desarrollo de estrategias educativas, resistencia a cambios educativos, incumplimiento en el proceso de toma de decisiones efectivas, entre otras que debilitan el sistema. (Gamboa, 2004).

Igualmente, Machado y Ramos (2005), agregan la escasa disponibilidad de los equipos y la procedencia de la información que se maneja y el comportamiento con los otros 
Tecnología de la información y comunicación como herramienta para la toma de decisiones en las instituciones educativas en Santa Marta, Colombia

Elvia Lacera Padilla - Marcos Orellana Parra - Gonzalo Gustavo Cortez (82:98)

integrantes del sistema educativo, situación ésta que se agrava aún más, considerando la poca disposición de las instituciones para ofrecer oportunidades para el manejo de tecnología. Dentro de este marco de ideas y gracias a observaciones de manera informal en instituciones educativas colombianas, también se pudo evidenciar la pérdida progresiva de la calidad en las unidades gerenciales educacionales, productos quizás de la desincorporación de herramientas tanto tecnológicas como comunicacionales.

Ahora bien, de acuerdo a observaciones asistemáticas por parte de la investigadora en las Instituciones Educativas Distritales en Santa Marta, Colombia, los directivos y muchos docentes no manejan información suficiente para el uso de las nuevas tecnologías en procesos gerenciales ni en el proceso educativo, solo utilizan la pizarra y la tiza, obviando los vídeos y teleconferencias, los chats, los foros, entre otros, donde además, no se toma en cuenta las etapas para la toma de decisiones en su esencia, vinculadas a la gestión educativa basada en las TIC para la formación del educador en el nuevo milenio.

Asimismo, se determinó que quienes integran las instituciones, en especial los gerentes educativos, no consideran el internet, el computador, el software educativo; entre otros, como herramientas para agilizar y reducir tiempos y espacios para las actividades de planificación y organización, así como también que el gerente educativo no promueve una gestión acorde con las nuevas tecnologías, obteniendo fracasos en los proyectos educativos, contrarias a lo que se reseña en el Plan de la Nación (2013-2018). Aunado a esto, se presentan constantes conflictos por no resolver las situaciones de una manera favorable.

De igual manera, se evidenció que existe debilidad en el papel que le corresponde asumir al personal de las instituciones, ante las alternativas educativas que se han implementado en los últimos años, lo cual implica cambiar hacia una actitud constructiva, de confianza en las potencialidades del docente; es decir, es necesario que el rol básico del gerente educativo sea el de investigar, analizar y facilitar el aprendizaje significativo, entre otros, para convertir al docente pasivo y callado en uno activo y consciente de su propia realidad. Es por ello, que de seguir esta situación, los empleados de las instituciones educativas pueden retrasar su proceso dinamizador del aprendizaje, lo cual traería a su vez inconvenientes en el desarrollo de estrategias innovadoras apegadas a los estándares más elevados de educación a niveles internacionales. Del mismo modo se perdería la calidad educativa dentro de las instituciones objeto de estudio.

Por lo antes planteado, surge la necesidad de desarrolla una investigación enfocada en el análisis en el uso de la tecnología de la información y la comunicación, como herramienta para la toma de decisiones en las instituciones educativas en Santa Marta, Colombia, con la finalidad de fomentar una transformación radical en el área educacional orientada al uso de las nuevas tecnologías de información y comunicación, que tanto para los directivos como los docentes ocupe un lugar destacado, con participación activa, fomente un compromiso ineludible de superación y competencia profesional, que les permita asumir retos y el mejoramiento en la toma de decisiones en los niveles directivos, impulsando una educación de calidad basado en sistemas innovadores en los aspectos gerenciales y educativos. 


\section{CONSIDERACIONES METODOLÓGICAS}

El artículo está enmarcado en una investigación tipo descriptiva, lo cual permitió determinar cómo el uso de la tecnología de la información y la comunicación es una herramienta para la toma de decisiones en las instituciones educativas en Santa Marta, Colombia, sin pretender establecer algún tipo de relación entre las variables. Considerando que el diseño de investigación es la estrategia empleada por el investigador para aproximarse al objeto de estudio, este trabajo se inserta en un diseño de campo, no experimental y transeccional.

En cuanto a la población de estudios, se tomó en cuenta las instituciones escolares públicas clasificadas dentro del rango de educación básica, específicamente de primaria, ubicadas en la ciudad de Santa Marta, clasificación emanada del Ministerio de Educación colombiano, donde el sistema educativo de ese país, lo conforman: la educación inicial, la educación preescolar, la educación básica (primaria cinco grados y secundaria cuatro grados), la educación media (dos grados y culmina con el título de bachiller) y la educación superior. (http://www.mineducacion.gov.co, 2018)

De manera que, a través de un muestreo no probabilístico incidental, se obtuvo como muestra las instituciones educativas agrupadas en la localidad 2, que cuentan con el Nivel Básico Primaria, ubicadas en la ciudad de Santa Marta, Colombia. De acuerdo a la Secretaría de Educación Distrital de Santa Marta estas ascienden a siete (7) instituciones educativas, las cuales se observan en detalle: IED Francisco de Paula Santander, IED Liceo Celeron, IED Técnico Industrial, IED Magdalena, IED Liceo el Saber, IED Liceo Samario. Sede 2 y Edgardo Vives Campos.

En virtud que las unidades educativas son fáciles de abordar se consideró realizar un censo, es decir se abordaron todos y cada uno de las unidades educativas que conforman la población. En este sentido se tomaron las siete (7) instituciones educativas a nivel de educación básica primaria pública, ubicada en la localidad 2, de la ciudad de Santa Marta, que a continuación se mencionan en virtud de su accesibilidad y en la disposición del personal directivo y docente de participar en la investigación.

Por otra parte, los sujetos o unidades informantes estuvo representados por los Rectores y el personal docente de las referidas instituciones, por ser estas personas las que cuentan con los conocimientos necesarios para suministrar la información requerida en la investigación. Según Parra (2006), la unidad de observación, es aquella por medio de la cual se obtiene la información; es decir, es la unidad informante. Quedando conformada por ochenta y seis (86) docentes y siete (7) rectores.

En la presente investigación se utilizó fuentes secundarias, para lo cual se realizó un arqueo biblo-hemerográfico para tomar las referencias necesarias para levantar la información teórica que sustentó la investigación. Por otra parte, también se emplearon fuentes primarias, para lo cual se diseñaron dos instrumentos de recolección de datos. El proceso de recolección 
de datos se realizó a través de la técnica de encuestas. El primer instrumento, dirigido a los Rectores, estuvo estructurado con 24 aseveraciones correspondientes a los indicadores planteados. El segundo instrumento fue aplicado a los 86 docentes de las instituciones seleccionadas. El mismo constó de 24 ítems que corresponden a los indicadores seleccionados para tal fin. Para ambos instrumento se utilizó una escala tipo Lickert con cinco alternativa de respuesta.

Estas cinco (5) alternativas de selección, se presentan a partir de valores mínimos (1) y máximos (5) de la escala presentada. Para establecer la escala de evaluación de los resultados, se procedió al cálculo del baremo. La interpretación de datos se efectuó por medio de un baremo, diseñado con miras a conocer la evaluación del indicador. Para ello se aplicó la siguiente fórmula:

$\mathrm{A}=0,80$

Donde:

A: Tamaño de clase (determina la amplitud de cada intervalo)

LM: Límite superior (5)

Lm: Límite inferior (1)

n: número de clases o alternativas en la escala de respuesta

El tamaño de la clase $(0,8)$ obtenido se sumó al valor de la primera escala de respuesta (1), resultando el primer intervalo (1-1,80), posteriormente se procedió a calcular el rango correspondiente a la segunda clase para lo cual se adicionó una centésima al valor máximo de la anterior para luego adicionar el tamaño de la clase, obteniéndose el rango de la segunda clase $(1,81-2,60)$. Este procedimiento se realizó para conformar el baremo, aplicándolo a todas las opciones de respuestas, hasta alcanzar la máxima puntuación posible. Este baremo fue utilizado para interpretar la media aritmética de los indicadores, dimensiones y la variable, permitiendo la interpretación de los resultados obtenidos.

Una vez diseñado los instrumentos a utilizar para la recolección de los datos, se debe garantizar la validez y la confiabilidad del mismo. En este sentido, se consultaron a cinco expertos para evaluar la correspondencia de los ítems planteados con respecto a las bases teóricas y a las variables de estudio planteadas, así como también a los objetivos expuestos. Las observaciones de los expertos fueron consideradas para fortalecer el instrumento de recolección de datos y así poder aplicarlo a la muestra seleccionada.

Al momento de comprobar la confiabilidad del instrumento, se aplicó una prueba piloto a treinta (30) sujetos. A través, de la mencionada prueba piloto se logró probar que el instrumento fue diseñado de una manera clara, sencilla y sobre todo comprensible por todos los miembros de la población estudiada, lo cual conlleva a un análisis de resultados más confiable y representativo de la realidad referida a las variables de estudio. Asimismo, los datos recolectados fueron procesados con el respaldo de un programa estadístico denominado SPSS versión 22, empleando el Coeficiente de Confiabilidad Alfa de Cronbach. 
El coeficiente de confiabilidad obtenido para el instrumento dirigido a los rectores fue de 0,852 y para el aplicado a los docentes de 0,899 . Indicando que ambos poseen una alta confiabilidad.

\section{PRESENTACIÓN Y DISCUSIÓN DE LOS RESULTADOS DEL ESTUDIO}

Para la discusión de los resultados, los datos se agrupan en tablas de frecuencias por indicadores y luego se presentan por dimensiones. De igual manera, se visualizan las medias para los indicadores y las dimensiones, las cuales permiten precisar si el uso de la tecnología de la información y la comunicación como herramienta para la toma de decisiones en las instituciones educativas en Santa Marta, Colombia se encuentran presentes o ausentes.

USO DE LAS TECNOLOGÍAS DE LA INFORMACIÓN Y COMUNICACIÓN (TIC)

Para Machado y Ramos (2005) y Cabero (20017), las TIC son un instrumento cada vez más indispensable en los centros educativos, abriendo nuevas posibilidades para la docencia como por ejemplo el acceso inmediato a nuevas fuentes de información y recursos (en el caso de Internet se puede utilizar buscadores), de igual manera el acceso a nuevos canales de comunicación (correo electrónico, Chat, foros...) que permiten intercambiar trabajos, ideas, información diversa, procesadores de texto, editores de imágenes, de páginas Web, presentaciones multimedia, utilización de aplicaciones interactivas para el aprendizaje: recursos en páginas Web, visitas virtuales. De manera que para, Cabero (2007), Cabero (2010), Machado y Ramos (2005) y Pere (2007), el uso de las TIC en las instituciones educativas se caracterizan por estar relacionadas con: el aprendizaje cooperativo, ser integradoras del currículo, por el fomento de una atención individualizada, como recurso de enseñanzaaprendizaje y como fuente de información.

En cuanto al aprendizaje cooperativo, Vélez (2012) y Machado y Ramos (2005), señalan que las Tecnologías de la Información y la Comunicación (TIC) motivan el aprendizaje cooperativo al permitir desarrollar destrezas y actividades cognitivas, creando nuevos ambientes educativos que despierten en el estudiante el deseo de aprender y que al relacionarlos con sus conocimientos previos puedan lograr un aprendizaje significativo a través de contenidos interesantes, dinámicos y divertidos.

En este sentido, los rectores $(42,9 \%)$ encuestados piensan que algunas veces los docentes manifiestan una actitud positiva hacia el aprendizaje cooperativo y algunas veces $(42,9 \%)$ se propicia la cooperación entre docentes en la aplicación de las TIC.

Por otra parte, hubo diferentes criterios de respuestas para el personal docentes, quienes opinaron que siempre $(52,4 \%)$, casi siempre $(26,2 \%)$ y algunas veces $(20,2 \%)$ ellos manifiestan una actitud positiva hacia el aprendizaje de las TIC y siempre (44\%), casi siempre (31\%) y algunas veces $(22,6 \%)$ manifiestan una orientación positiva hacia las TIC y piensan que en la institución se propicia la cooperación entre ellos en la aplicación de las TIC. 
Tecnología de la información y comunicación como herramienta para la toma de decisiones en las instituciones educativas en Santa Marta, Colombia

Elvia Lacera Padilla - Marcos Orellana Parra - Gonzalo Gustavo Cortez (82:98)

Al señalar a las TIC como Integradoras del currículo, Machado y Ramos (2005), argumentan que las TIC deben tener como uso la integración del currículo, para lo cual debe llevarse a cabo a través de un proceso cuidadoso, y cumpliendo una serie de condiciones y etapas para que puedan ya que de no ser así, puede se puede hacer una mala integración, o peor aún, ni siquiera integrarlas realmente, sino simplemente adquirir equipos y adicionar una asignatura más que los alumnos y profesores ven como materia de estudio, es decir, se puede confundir la integración tecnológica con la enseñanza solo de computación o informática, donde se lleva a alumnos una veces por semana a la sala de informática a manejar programas.

La situación presentada, con respecto al indicador antes mencionado, en las instituciones escolares abordadas, se describe, al entrevistar a los Rectores, para quienes algunas veces $(57,1 \%)$, se usa las TIC para apoyar los objetivos curriculares. En el mismo sentido, el personal docente difiere de la opinión de los Rectores, donde el 39,3\% expresó que algunas veces se le da ese uso a las TIC, mientras que $32,1 \%$ señaló que siempre y el $23,8 \%$ casi siempre. Po otra parte, un alto porcentaje de Rectores manifestó que siempre $(71,4 \%)$, en la institución se utilizan herramientas tecnológicas, como recurso para la adquisición de conocimientos. En tanto para los docentes siempre $(46,4 \%)$, se le da ese uso a la TIC.

Al puntualizar el indicador: Atención Individualizada, Cabero (2007), argumenta que cuando los estudiantes utilicen la herramienta de las TIC en el proceso de enseñanza - aprendizaje requieren una atención especialmente individualizada, hasta tanto adquiera habilidades y destrezas que le permita autodirigir su proceso según sus intereses y necesidades. $\mathrm{Al}$ inicio, el docente debe actuar como facilitador y luego dejar al alumno en libertad para desarrollar las actividades según sus necesidades personales.

En este sentido, la situación presentada en las instituciones educativas abordadas, indica que la mayoría de los Rectores $(57,1 \%)$ piensan que algunas veces en la institución las TIC se usan para brindar atención personalizada a los estudiantes; mientras que el 28,6\% de los mismos argumentó que casi nunca se le daba ese uso. Semejante comportamiento presentan las respuestas dada por los docentes, quienes en su mayoría piensan que algunas veces $(46,4 \%)$ usan las TIC para brindar atención personalizada. El resto se ubicó en las alternativas siempre $(17,9 \%)$, casi siempre $(15,5 \%)$ y casi nunca $(16,7 \%)$. En el mismo sentido, se mantiene la tendencia de la mayoría de los rectores en cuanto a su apreciación poco favorable, en cuanto a si los docentes fomentan nuevos ambientes educativos que despiertan en el estudiante el deseo de aprender. Coincidiendo los docentes con las respuestas dadas por los rectores, para quienes casi siempre $(47,6 \%)$ casi siempre $(26,2 \%)$ y siempre $(22,6 \%)$ ellos fomentan nuevos ambientes educativos.

Al señalar a las TIC como recursos de enseñanza- aprendizaje, Cacheiro (2011), indica que la utilización de las TIC compromete al docente a estar actualizado con respecto a las nuevas tecnologías de la información y la comunicación, él debe conocer las innovaciones, así como también utilizarlas y promoverlas a fin de ser empleadas como verdaderos recursos de aprendizaje. Le permite al docente pasar de un uso informativo y colaborativo a un uso didáctico para lograr unos resultados de aprendizaje. 
Dadas estas consideraciones se evidencia que más de la mitad de los Rectores (57,1\%) aseguran que algunas veces los docentes usan las TIC para facilitar la adaptación a distintos ritmos de aprendizaje de los alumnos. Y un 28,6\% piensa que casi nunca le dan tal uso. Al respecto, los docentes coinciden en afirmar que algunas veces $(58,3 \%)$ usan las TIC con ese propósito, en tanto otro grupo se ubicó en la alternativa casi siempre $(19,1 \%)$, siempre $(14,3 \%)$ y casi nunca $(13,1 \%)$. Por otra parte, se observa una tendencia distinta de las respuestas de los Rectores, en cuanto a si en la unidad escolar se cuenta con planes de desarrollo institucional para la implantación de las TIC como herramienta pedagógica; ellos indicaron que siempre $(57,1 \%)$ se les da ese uso. En contraposición, los docentes entrevistados aseguran que algunas veces $(40,5 \%)$ y casi nunca $(20,2 \%)$ la implantación de las TIC como herramienta pedagógica obedecen a planes de desarrollo institucional.

Para finalizar con el uso de las TIC en las instituciones escolares estudiadas, Cabero (2007) y Pere (2007), coinciden en afirmar que emplear las TIC como fuentes de información permiten la interacción entre estudiantes y docentes donde pueden intercambiar experiencias enriquecedoras del aprendizaje con otros grupos que desarrollen procesos de formación similar o diferente. Al respecto, más de la mitad de los Rectores $(57,1 \%)$ tiene una opinión desfavorable con respecto a si los docentes emplean activamente las aplicaciones tecnológicas y trabajan en red con otras escuelas, empresas y el sector gubernamental. Sin embargo, hay respuestas diversas entre el grupo de docentes; ya que para el $42,9 \%$ de ellos, algunas veces emplean estas aplicaciones tecnológicas; igual porcentaje piensa que casi siempre (20,2\%) y casi nunca $(20,2 \%)$ esto ocurre y solo $11,9 \%$ dio como alternativa de respuesta siempre.

Nótese la orientación positiva de las respuestas dadas por los Rectores entrevistados en cuanto a si se usan las TIC como fuente de información de apoyo a las actividades institucionales, éstos afirmaron que siempre $(57,1 \%)$ y casi siempre $(42,9 \%)$ se usan las TIC en este sentido. En este marco de ideas, la mayor parte de los docentes también dieron respuestas favorables, ubicándose en las alternativas siempre $(42,9 \%)$, casi siempre $(38,1 \%)$ $\mathrm{y}$ algunas veces $(19 \%)$.

\section{NECESIDADES DE CAPACITACIÓN DEL PERSONAL DOCENTE EN EL USO DE LAS TECNOLOGÍAS DE LA INFORMACIÓN Y COMUNICACIÓN (TIC)}

Para Valdés y col. (2011) la capacitación del docente en el uso y manejo de herramientas de la tecnología de la información y comunicación, amerita educadores creativos que transformen los ambientes de aprendizaje, para facilitar al alumno el proceso de enseñanzaaprendizaje. Las TIC facilitan la práctica del personal docente quienes al desarrollar competencias adecuadas en el uso de las mismas la pueden utilizar como apoyo a la enseñanza y el aprendizaje, donde la interacción con los estudiantes se hace más dinámica y atractiva. 
Tecnología de la información y comunicación como herramienta para la toma de decisiones en las instituciones educativas en Santa Marta, Colombia

Elvia Lacera Padilla - Marcos Orellana Parra - Gonzalo Gustavo Cortez (82:98)

En este sentido, Rosario y Vásquez (2012) y Valdez y col. (2011), coinciden en señalar que el nuevo entorno en el cual se desenvuelve la escuela, requiere la presencia de las TIC en la formación y capacitación de los docentes. Estos deben poseer nuevas capacidades que le permitan alinearse a las demandas sociales y a los intereses de los estudiantes. Ya no es suficiente desarrollar contenidos de manera tradicional, se requiere que los docentes se involucren en la elaboración y desarrollo de los modelos didácticos, que permitan realizar las actividades de manera diferentes y favorecer la creación de entornos más ricos, interactivos y variados donde la participación del alumno es imprescindible.

De manera que en los resultados se evidencian respuestas favorables; donde los Rectores (57,1\% siempre y $42,9 \%$ casi siempre), argumentan que en la institución se plantea las necesidades de formación de los docentes con la intención de generar la incorporación de las TIC al proceso enseñanza-aprendizaje. Similar comportamiento tiene las respuestas dadas por docentes, donde un $45,2 \%$ señaló casi siempre y un 33,3\% opinó que siempre se plantean estas necesidades de formación dentro de la institución. De igual manera, los Rectores (42, $9 \%$ siempre y $42,9 \%$ algunas veces) expresaron que en la institución se propicia la innovación y el desarrollo en los programas de formación basado en las TIC. En tanto, para el 40, 5\% y $33,3 \%$ de los docentes casi siempre y siempre respectivamente, se genera tal iniciativa.

De manera que, la formación del personal y el diseñar estrategias que minimicen la resistencia al uso de las TIC, son requisitos fundamentales que debe impulsar la institución educativa, a fin de potenciar la práctica docente, y de esta manera contribuir a la mejora de la calidad de la educación y la propia formación del docente, con mira a los requerimientos de una sociedad basada en el conocimiento.

En cuanto a la resistencia al cambio en el uso de las TIC, Paredes (2004) comenta que para comprender el cambio y las resistencias al cambio se hace necesario entender las fuerzas que operan en el seno de los centros educativos y la forma en que construyen sus normas. Estas normas tienen que ver con el papel de los diferentes actores de los centros en la adopción de fines de los mismos, la mejora continua o el apoyo mutuo, pero también otras normas como el respeto a la individualidad y la creatividad, normas por las cuales las instituciones educativas sostienen una forma de hacer. Señala que el conocimiento que poseen los docentes sobre el uso correcto de las TIC puede ser limitado y presentar inconsistencias y por tanto resistencia a su uso, ya que a algunos les resulta difícil innovar y estar abiertos a nuevas ideas y procedimientos, de allí que se torna imperativo reforzar la formación y la provisión de información específica donde se presente como una herramienta que coadyuva a la mejora continua y a la creatividad, lo cual apunta a potenciar del proceso enseñanzaaprendizaje.

Las respuestas dadas por la población abordada, permite observar que un grupo significativo de Rectores piensan que casi siempre $(71,4 \%)$ y siempre $(28,6 \%)$ los docentes 
se sienten a gusto trabajando con las TIC, mientras que el 48,8\% de los docentes expresó que siempre y el $38,1 \%$ casi siempre esto es así. Por otra parte, para los Rectores entrevistados, los docentes se adaptan rápidamente a los cambios para enfrentar los retos que plantea el entorno con respecto al uso de la TIC. Opinión con orientación positiva respaldada por el personal docente, quienes más de la mitad de la muestra respondió con la alternativa siempre (44\%) y casi siempre (40,5\%). Estos resultados permiten inferir, que los docentes no presentan resistencia al cambio con respecto a la utilización de las TIC y en la institución se hacen esfuerzos para formarlos en estas capacidades.

Como cierre a la discusión presentada, en tabla 1 se observan los resultados de la variable Tecnologías de la Información y Comunicación(TIC), identificándose las frecuencias de las respuestas para las dos dimensiones: Uso de las Tecnologías de la Información y Comunicación y necesidades de capacitación del personal docente. En ambas se visualiza la misma tendencia favorable para ambos segmentos poblacionales. La mayor frecuencia de las respuestas de los rectores estuvo enmarcada en la escala siempre (35\%) y el menor porcentaje en casi nunca $(8,9 \%)$. De igual manera para la muestra de docentes la alternativa siempre $(34,6 \%)$ fue la que alcanzó más alto porcentaje y el menor en nunca $(0,6 \%)$. Además se visualizan los promedios ponderados alcanzando niveles que permiten inferir presencia de las TIC en las instituciones escolares abordadas, sin embargo se deben tomar acciones que apunten a mejorar estos resultados debido a que los promedios están muy cerca de extremo inferior de la media.

Tabla 1. Tecnologías de la Información y Comunicación (TIC)

\begin{tabular}{|c|c|c|c|c|c|c|c|c|c|c|c|c|}
\hline Muestra & \multicolumn{5}{|c|}{ Rectores } & \multicolumn{5}{|c|}{ Docentes } & \multicolumn{2}{|c|}{ Media } \\
\hline $\begin{array}{l}\text { Alternativas } \\
\qquad \text { Dimensiones }\end{array}$ & $\underset{\%}{\text { Siempre }}$ & $\begin{array}{c}\text { Casi } \\
\text { siempre } \\
\%\end{array}$ & $\begin{array}{c}\text { Algunas } \\
\text { veces } \\
\%\end{array}$ & $\begin{array}{c}\text { Casi } \\
\text { nunca } \\
\%\end{array}$ & $\underset{\%}{\text { Nunca }}$ & $\underset{\%}{\text { Siempre }}$ & $\begin{array}{c}\text { Casi } \\
\text { siempre } \\
\%\end{array}$ & $\begin{array}{c}\text { Algunas } \\
\text { veces } \\
\%\end{array}$ & $\begin{array}{c}\text { Casi } \\
\text { nunca } \\
\%\end{array}$ & $\underset{\%}{\text { Nunca }}$ & Rector & Doc. \\
\hline $\begin{array}{l}\text { Uso de las } \\
\text { Tecnologías de } \\
\text { la Información y } \\
\text { Comunicación } \\
\end{array}$ & 27.1 & 18.6 & 40.0 & 14.3 & - & 29.4 & 25.3 & 36.1 & 8.1 & 1.1 & 3.59 & 3.75 \\
\hline $\begin{array}{l}\text { Necesidades de } \\
\text { capacitación del } \\
\text { personal docente }\end{array}$ & 42.8 & 32.2 & 21.4 & 3.6 & - & 39.8 & 41.1 & 18.2 & 0.9 & - & 4.15 & 4.01 \\
\hline$\%$ Variable & 35.0 & 25.4 & 30.7 & 8.9 & - & 34.6 & 33.2 & 27,1 & 4.5 & 0.6 & & \\
\hline \multicolumn{11}{|c|}{ Media de la variable. Rectores/ Docentes } & 3.87 & 3.88 \\
\hline
\end{tabular}

Fuente: Elaboración propia

\section{TIPOS DE TOMA DE DECISIONES EN LAS INSTITUCIONES ESCOLARES}

En la sección que se presentan a continuación, se detallan los resultados obtenidos para la segunda variable abordada en esta investigación: Toma de decisiones. Aquí se consideran: Tipos de toma de decisiones y características de la toma de decisiones, así como los indicadores y las aseveraciones que se desprenden de ellos. 
Tecnología de la información y comunicación como herramienta para la toma de decisiones en las instituciones educativas en Santa Marta, Colombia

Elvia Lacera Padilla - Marcos Orellana Parra - Gonzalo Gustavo Cortez (82:98)

Una de las responsabilidades más importantes en una institución es la toma de decisiones. Münch y García (2012), argumentan que esta responsabilidad le permite a través de una acertada o no selección de alternativas el éxito o fracaso de los objetivos propuestos. De manera que la toma de decisiones afecta el desempeño de los docentes y la satisfacción laboral de los mismos. Aquí se consideran la toma de decisiones programadas y las no programadas.

Carrión (2007) y Hitt y col (2006), señalan que las decisiones programadas son las rutinarias, con alto grado de certidumbre, donde la organización desarrolla procedimientos formales para decidir sobre ellos. Dado esto, el director de las instituciones escolares debe comprender claramente la naturaleza del problema para poderlo definir y plantear las posibles soluciones.

A continuación, se exhiben los resultados. Nótese como la mayoría de las respuestas dadas para, tanto por los Rectores como por los docentes, se ubican en la alternativa algunas veces. Así para el 71,4\% de los rectores, algunas veces en la institución se utilizan las TIC para detectar las necesidades de forma precisa. De igual manera un 50\% del personal docente señaló que algunas veces se utilizan las TIC para detectar las necesidades institucionales. En tanto las respuestas del otro $50 \%$ de los docentes estuvieron repartidas entre las alternativas casi siempre $(26,2 \%)$, siempre $(16,7 \%)$ y casi nunca $(7,1 \%)$.

Por otra parte, un grupo de Rectores argumentó que algunas veces $(42,9 \%)$, casi siempre $(28,5 \%)$, siempre $(14,3 \%)$ y casi nunca $(14,3 \%)$, en la institución se desarrollan procedimientos formales, apoyados en las TIC para la solución de problemas. Por su parte, el 45,2\% (algunas veces), el 31,3\% (casi siempre) y el 19\% (siempre) de los docentes corroboraron esta posición de los Rectores. Solo un menor porcentaje asumió una posición negativa al respecto.

$\mathrm{Al}$ referirse a las decisiones no programadas, éstas se toman a nivel de alta gerencia y son aquellas recientes y sin estructurar, por lo tanto no existe un procedimiento establecido para el abordaje del problema, se manejan como procesos generales, juicios, intuición y creatividad (Carrión, 2007; Hitt y col. 2006). En este sentido, en las instituciones educativas los directivos necesita un buen criterio para tomar buenas decisiones y esta capacidad implica por sí misma, una combinación de conocimientos, experiencias, valores y destrezas que puedan señalar un curso de acción que no sea necesariamente producto de un razonamiento estrictamente lógico. No cabe duda que la capacidad de razonar sistemáticamente es una necesidad básica para cualquier directivo. Los que tienen éxito, han desarrollado esta capacidad a través de la experiencia.

De manera que en los resultados se observa que tanto los Rectores $(42,8 \%)$ como el personal docente $(47,6 \%)$ argumentan que algunas veces el uso de las TIC les facilita tomar decisiones novedosas y desestructuradas. Para el resto de los rectores siempre $(28,6 \%)$ y casi siempre $(28,6 \%)$ esto es así. En tanto para los docentes el 31\% (casi siempre) y el 11,9\% (siempre) afirmó que el uso de la TIC facilita a los Rectores este tipo de toma de decisiones. 


\section{CARACTERÍSTICAS DE LA TOMA DE DECISIONES}

Cuanto más complejo sea el problema, más desafiante serán tanto la decisión como su implementación eficaz, de allí que las características del proceso de decisión dependen de la situación que lo genera, por tanto se hace necesario que el personal directivo asuma sus responsabilidades en la adecuada selección de alternativas de decisión para resolver las situaciones generadas a lo interno de la institución ya que de las buenas o malas decisiones va a depender el éxito institucional.

Las características personales de quien toma la decisión es el primer aspecto que según Hitt y col (2006) coadyuva a que las decisiones son sean tan objetivas y racionales, debido a la carga valorativa de los sujetos que ocupan cargos directivos, así como la visión que posean de la realidad. Por otra parte, pueden verse limitados por su capacidad cognitiva de las herramientas requeridas para obtener y procesar la información requerida para tomar las decisiones acertadas.

Los resultados hacen referencia a las características de quien toma la decisión, donde los Rectores manifestaron que en la institución casi siempre $(57,1 \%)$ y siempre $(42,9 \%)$ se tiende a tomar la primera solución posible cuando se le presenta una dificultad. Mientras que los docentes piensan que casi siempre (47,6\%) y siempre $(34,5 \%)$ el Rector manifiesta esta tendencia ante las dificultades. Esta respuesta puede interpretarse como que los Rectores no evalúan otras alternativas de solución que los lleve a enfrentar el problema de manera más certera.

Por otra parte los Rectores opinaros que siempre $(85,7 \%)$ usan las TIC para no sentirse abrumado por la cantidad de información que necesitan procesar para apoyar los objetivos curriculares. Opiniones diversas personal docente entrevistado, donde el 36,9\% seleccionó la alternativa siempre, el $32,1 \%$ casi siempre y $25 \%$ algunas veces.

En cuanto a las características del problema, se observan las respuestas dadas con orientación positiva tanto por los Rectores como por los docentes. Así se tiene, que igual porcentaje de Rectores piensa que siempre $(28,6 \%$ ) y casi siempre $(28,6 \%)$, se cuenta con la información necesaria suministrada por las TIC para la solución de los problemas planteados, mientras que un $42,9 \%$ expresó que algunas veces esto es así. Por otra parte, los docentes piensan que casi siempre $(45,2 \%)$, algunas veces $(28,6 \%)$ y siempre $(26,2 \%)$ los Rectores solucionan los problemas planteados a partir de la información suministrada por las TIC. Mientras que un alto porcentaje de Rectores $(85,7 \%)$ y de docentes $(59,5 \%)$ afirmaron que casi siempre se cuentan con estrategias alternativas para tomar decisiones, en caso de imprevistos. 
Tecnología de la información y comunicación como herramienta para la toma de decisiones en las instituciones educativas en Santa Marta, Colombia

Elvia Lacera Padilla - Marcos Orellana Parra - Gonzalo Gustavo Cortez (82:98)

En otro orden de ideas, las características del ambiente influyen en la decisión, de allí que tomar la decisión bajo la presión de tiempo considerable, las limitaciones de los recursos, la importancia del problema y la magnitud de la decisión se convierten en algunos de los aspectos que se deben considerar al tomar una decisión ( Hitt y col. 2006).

En este sentido, la situación presentada en las instituciones educativas abordadas, permiten señalar que la mayoría de los Rectores (siempre $57,1 \%$ y casi siempre $42,9 \%$ ) se identifican con los problemas de la incorporación de las TIC a la institución, visualizando el entorno donde se desarrolla. Mientras que el $60,7 \%$ de los docentes argumentó que casi siempre, el $21,4 \%$ siempre y el $17,9 \%$ que algunas veces se presenta esta situación. En el mismo orden de ideas, más de la mitad de la muestra de Rectores señaló que siempre $(57,1 \%)$ y casi siempre $(42,9 \%)$ se toman en cuenta los recursos disponibles para tomar las decisiones sobre la incorporación de las TIC a la institución. En tanto, que los docentes piensan que casi siempre $(54,8 \%)$, siempre $(29,8 \%)$ y algunas veces $(15,5 \%)$, se consideran los recursos disponibles.

Ya para finalizar con la presentación de los resultados, en la tabla 2 exhibe el comportamiento de las respuestas, tanto para Rectores como para docentes, de la variable toma de decisiones; donde la alternativa siempre tiene el mayor porcentaje $(34,6 \%)$ para el grupo de Rectores; mientras que para los docentes las respuestas estuvo orientada hacia la alternativa casi siempre $(41,2 \%)$. Los valores negativos mantuvieron una baja presencia en las respuestas dadas. De igual manera los promedios ponderados dan cuenta de la presencia de la toma de decisiones en las instituciones abordadas.

Tabla 2. Toma de decisiones

\begin{tabular}{|c|c|c|c|c|c|c|c|c|c|c|c|c|}
\hline Muestra & \multicolumn{5}{|c|}{ Rectores } & \multicolumn{5}{|c|}{ Docentes } & \multicolumn{2}{|c|}{ Media } \\
\hline $\begin{array}{l}\text { Alternativas } \\
\text { Dimensiones }\end{array}$ & $\underset{\%}{\text { Siempre }}$ & $\begin{array}{c}\text { Casi } \\
\text { siempre } \\
\%\end{array}$ & $\begin{array}{c}\text { Algunas } \\
\text { veces } \\
\%\end{array}$ & $\begin{array}{c}\text { Casi } \\
\text { nunca } \\
\%\end{array}$ & $\underset{\%}{\operatorname{Nunca}}$ & $\underset{\%}{\text { Siempre }}$ & $\begin{array}{c}\text { Casi } \\
\text { siempre } \\
\%\end{array}$ & $\begin{array}{c}\text { Algunas } \\
\text { veces } \\
\%\end{array}$ & $\begin{array}{c}\text { Casi } \\
\text { nunca } \\
\%\end{array}$ & $\underset{\%}{\text { Nunca }}$ & Rector & Doc. \\
\hline $\begin{array}{c}\text { Tipos de toma de } \\
\text { decisiones }\end{array}$ & 21.5 & 25.0 & 49.9 & 3.6 & - & 22.6 & 32.4 & 25.1 & 12.0 & 7.9 & 3.65 & 3.65 \\
\hline $\begin{array}{l}\text { Características } \\
\text { de la toma de } \\
\text { decisiones }\end{array}$ & 47.6 & 42.9 & 7.1 & - & 2.4 & 27.3 & 49.9 & 21.8 & 0,8 & 0.2 & 4.33 & 4.03 \\
\hline$\%$ Variable & 34.6 & 33.9 & 28.5 & 1.8 & 1.2 & 24.9 & 41.2 & 23.4 & 6,4 & 4.1 & & \\
\hline \multicolumn{11}{|l|}{$\because \quad$ I } & 3.99 & 3.84 \\
\hline
\end{tabular}

Fuente: Elaboración propia 


\section{CONCLUSIÓN}

Dado los resultados para ambas variables, se puntualiza que la Tecnología de la Información y Comunicación se encuentra presente como herramienta en la Toma de decisiones, en las siete (7) instituciones educativas agrupadas en la localidad 2 de Santa Marta. Sin embargo, estos valores se encuentran muy cercanos al extremo inferior de media ponderada, lo cual alerta a prestar especial atención ya que puede ser susceptible a descender a valores inferiores.

Se invita a los rectores y docentes a considerar que en gran medida, el éxito de la organización escolar depende de la calidad de las decisiones que tomen sus administradores, para lo cual se requiere del procesamiento de una gran cantidad de información. En este contexto existen las tecnologías de la información y comunicación (TIC) que le dan soporte a la toma de decisiones. En este sentido, se puede afirmar que la toma de decisiones en las instituciones abordadas constituye un proceso informacional que se desarrolla por las personas que interactúan en el quehacer académico, para solucionar problemas y aprovechar las oportunidades del entorno.

\section{REFERENCIAS BIBLIOGRÁFICAS}

- Aguerrondo, I. (Compil) (2006) La integración de las Tecnologías de la Información y la Comunicación en los Sistemas Educativos. Estado del arte y orientaciones estratégicas para la definición de políticas educativas en el sector. IIPE- UNESCOSede Regional Buenos Aires, Argentina. Documento en línea Disponible en: unesdoc.unesco.org.pdf. Consulta: 28/05/2017

- Cabero, J. (2007). Las necesidades de las TIC en el ámbito educativo: oportunidades, riesgos y necesidades. En Revista Tecnología y Comunicación Educativa. Año 21, N45. Pp 4-19 Julio-diciembre. Documento en línea Disponible en: http:// investigacion.ilce.edu.mx/tyce/45/ Consulta: 10/06/2017

- Cabero, J. (2010). Los retos de la integración de las TICs en los procesos educativos. Límites y posibilidades. En Revista Perspectiva Educacional, Formación de Profesores. Recuperado: http://4www.redalyc.org/articulo.oa.10/03/2017.

- Cacheiro, M. (2011). Recursos Educativos TIC de Información, Colaboración y Aprendizaje. En Píxel-Bit. Revista de Medios y Educación No 39. Pp. 69-81. España. Disponible en: acdc.sav.us.es/pixelbit/images/stories/p39/06.pdf Consulta: 12/06/2017.

- Carrión, J. (2007). Estrategia. De la Visión a la Acción. Segunda edición. España. ESISC Editorial Alfaomega.

- Gamboa, S. (2004). Creatividad y entornos virtuales de aprendizaje. Primera edición. Colombia Editorial Universidad Pedagógica Nacional.

- Hitt, M., Black, S. y Porter, L. (2006). Administración. Novena edición. México. Pearson Educación.

- Machado L. y Ramos, F. (2005). TIC Una Propuesta Metodológica de Integración Tecnológica al Currículo. Colombia. Editorial Universidad Pedagógica Nacional. 
Tecnología de la información y comunicación como herramienta para la toma de decisiones en las instituciones educativas en Santa Marta, Colombia

Elvia Lacera Padilla - Marcos Orellana Parra - Gonzalo Gustavo Cortez (82:98)

- Münch, L. y García, J. (2012). Fundamentos de Administración. México. Editorial Trillas

- Paredes, J. (2004). Cultura escolar y resistencias al cambio en Educación Secundaria. Tendencias pedagógicas. En Revista del Departamento de Didáctica y Teoría de la Educación de la Universidad Autónoma de Madrid, Nª, pp. 131-142. Disponible en: https://www.uam.es/personal_pdi/stmaria/jparedes/practica/paredes_04_01. pdf Consulta: 26/06/2017

- Patru M. y Semenov, A. (2005). Las tecnologías de la información y la comunicación en la enseñanza. Manual para docentes o Cómo crear nuevos entornos de aprendizaje abierto por medio de las TIC. Editado por la UNESCO. Montevideo, Uruguay. Disponible en: unesdoc.unesco.org/images/0013/001390/139028s.pdf. Consulta: $16 / 05 / 2017$

- Pere. G. (2007). Impactos de la TIC en educación: Funciones y limitaciones. Departamento de Pedagogía Aplicada, Facultad de Educación, Universidad de Barcelona. España. Disponible en: http://dewey.uab.es/pmarques/siyedu.htm Consulta: 15-07-17

- Parra, J. (2006). Guía de Muestreo. Segunda edición. Venezuela. Facultad de Ciencias Económicas y Sociales de la Universidad del Zulia. Colección XLV aniversario FCES.

- República de Colombia. Ministerio de Educación. Disponible en: http://www. mineducacion.gov.co. Consulta: 12/02/2018.

- República de Colombia. Alcaldía de Santa Marta. Secretaría de Educación. Disponible en www.educacion.santamarta.gov.co/colegios/2018 Consulta: 12/02/2018.

- Rosario, H. y Vásquez, L. (2012). Formación del Docente Universitario en el uso de TIC. Caso Universidades Públicas y Privadas. (U. De Carabobo y U. Metropolitana). En Píxel-Bit. Revista de Medios y Educación. № 41. Pp163-171. Disponible en: acdc.sav.us.es/pixelbit/images/stories/p39/06.pdf. Consulta: 12/06/2017.

- UNESCO (2009). Tras la pista de una revolución académica: Informe sobre las tendencias actuales para la Conferencia Mundial sobre la Educación Superior. Disponible en: http://unesdoc.unesco.org. Consulta: 16/05/2017

- UNESCO (2013). Enfoques estratégicos sobre las TICs en Educación en América Latina y el Caribe. Oficina Regional de Educación para América Latina y el Caribe (OREALC/UNESCO Santiago).Chile. Disponible en:www.unesco.org/new/ fileadmin/MULTIMEDIA/FIELD/Santiago.pdf. Consulta: 16/05/2017

- Valdés A., Angulo J., Urias, M., García, R. y Mortis, S. (2011). Necesidades de capacitación de docentes de Educación Básica en el uso de las TIC. En Pixel-Bit. Revista de Medios y Educación. Disponible en http://www.redalyc.org/articulo.oa. Consulta: $10 / 03 / 2017$

- Vélez, C. (2012). Estrategias de enseñanza con uso de las Tecnologías de la Información y Comunicación para favorecer el trabajo significativo. Trabajo de Grado de Maestría en Tecnología Educativa y Medios Innovadores para la Educación. Tecnológico de Monterrey. México. Disponible en::https://es.scribd. $\mathrm{com} / . . /$ Tesis-estrategias-de-ensenanza-con-uso-de-las-tecnologias Consulta: $25 / 06 / 2017$ 